\title{
Attenuation of Porphyromonas gingivalis oral infection by $\alpha$-amylase and pentamidine
}

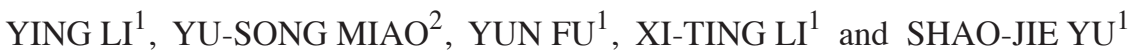 \\ ${ }^{1}$ Department of Periodontology, Guanghua School of Stomatology, Sun Yat-sen University; \\ ${ }^{2}$ Department of Dental Science, Guangzhou Chest Hospital, Guangzhou, Guangdong 510055, P.R. China
}

Received November 18, 2013; Accepted July 4, 2014

DOI: $10.3892 / \mathrm{mmr} .2015 .3584$

\begin{abstract}
The Porphyromonas gingivalis bacterium is one of the most influential pathogens in oral infections. In the current study, the antimicrobial activity of $\alpha$-amylase and pentamidine against Porphyromonas gingivalis was evaluated. Their in vitro inhibitory activity was investigated with the agar overlay technique, and the minimal inhibitory and bactericidal concentrations were determined. Using the bactericidal concentration, the antimicrobial actions of the inhibitors were investigated. In the present study, multiple techniques were utilized, including scanning electron microscopy (SEM), general structural analysis and differential gene expression analysis. The results obtained from SEM and bactericidal analysis indicated a notable observation; the pentamidine and $\alpha$-amylase treatment destroyed the structure of the bacterial cell membranes, which led to cell death. These results were used to further explore these inhibitors and the mechanisms by which they act. Downregulated expression levels were observed for a number of genes coding for hemagglutinins and gingipains, and various genes involved in hemin uptake, chromosome replication and energy production. However, the expression levels of genes associated with iron storage and oxidative stress were upregulated by $\alpha$-amylase and pentamidine. A greater effect was noted in response to pentamidine treatment. The results of the present study demonstrate promising therapeutic potential for $\alpha$-amylases and pentamidine. These molecules have the potential to be used to develop novel drugs and broaden the availability of pharmacological tools for the attenuation of oral infections caused by Porphyromonas gingivalis.
\end{abstract}

Correspondence to: Dr Ying Li, Department of Periodontology, Guanghua School of Stomatology, Sun Yat-sen University, 56 Lingyuan West Road, Guangzhou, Guangdong 510055, P.R. China

E-mail: yingli142@gmail.com

Key words: Porphyromonas gingivalis, antimicrobial, $\alpha$-amylase, pentamidine

\section{Introduction}

Porphyromonas gingivalis ( $P$. gingivalis) is a Gram-negative, rod-shaped, anaerobic pathogenic bacterium associated with several periodontal diseases (1). The occurrence of periodontitis in $>47 \%$ of the US population, with a high prevalence of mild $(8.7 \%)$, moderate $(30 \%)$ and severe $(8.5 \%)$ cases, is due to variables, such as oral hygiene, socioeconomic status and other environmental, genetic and metabolic risk factors (2). $P$. gingivalis exhibits a strong positive association with the diagnostic parameters of periodontitis, including gingival recession, increased sulcular pocket depth and bleeding upon probing (3). In addition, Hajishengallis et al (4) demonstrated that although $P$. gingivalis does not independently cause periodontal disease in a germ-free murine model, low numbers of $P$. gingivalis are able to disrupt host homeostasis through actions involving commensal microorganisms and complement, leading to inflammation and periodontal disease (4).

$P$. gingivalis produces multiple virulence factors that allow successful colonization and support evasion of host defenses, a number of which contribute to the inflammation and destruction of host tissues (5). Adhesins, such as fimbraie and hemagglutinins, promote attachment $(5,6)$ and proteolytic enzymes, such as cysteine proteinases and hemagglutinins, are capable of degrading multiple substrates in the gingival crevice, facilitating nutrient acquisition and contributing to host tissue degradation $(5,6)$.

The control of oral bacteria is mediated by a diverse array of specific and non-specific innate immune molecules present in saliva and on mucosal surfaces (7). There are number of functional families consisting of $>45$ antimicrobial proteins and peptides, including cationic peptides, metal ion chelators, histatins, defensins, bacterial adhesions and agglutinators and enzymes directed at the bacterial cell wall. However, the physiological concentration of the majority of salivary antimicrobial proteins and peptides is lower than the effective concentration in vivo (7), which suggests that there may be additional immune functions within the saliva.

The enzyme $\alpha$-amylase catalyzes the hydrolysis of internal $\alpha-1,4$-glycosidic linkages within carbohydrate moieties, including glucose, maltose and maltotriose units $(8,9)$. $\alpha$-amylases are used in a number of industrial processes in the food, fermentation, textiles, paper, detergent and pharmaceutical industries. Fungal and bacterial amylases may have 
the potential for use in the pharmaceutical and fine-chemical industries. Advances in biotechnology have led to the expansion of amylase application in numerous fields, such as biomedical and analytical chemistry, and also textiles, food, brewing and distilling industries $(8,10)$.

The bisbenzamidine derivative, pentamidine, has proved one of the most successful agents for targeting eukaryotic parasites, and has been used clinically for $>70$ years $(11,12)$. In 1938, pentamidine isethionate was identified to have antiprotozoal activity, and was approved in the United States for the treatment of Pneumocystis carinii pneumonia and other protozoal diseases (13). Pentamidine has the ability to inhibit interaction at the $\mathrm{Ca}^{2+} / \mathrm{p} 53$ site of the protein, and has been reported to inhibit $\mathrm{S} 100 \mathrm{~B}$ activity (14). On the basis of the previous studies, the present study aimed to take advantage of the antimicrobial activity of $\alpha$-amylase and pentamidine to attenuate oral infection of $P$. gingivalis.

\section{Materials and methods}

Reagents and chemicals. $\alpha$-amylase from porcine pancreas was purchased from Sigma-Aldrich (St. Louis, MO, USA). The bisbenzamidine derivative, pentamidine was purchased from Sanofi S.A. (Paris, France). The media (Terrific broth and Luria-Bertani broth) were obtained from Thermo Fisher Scientific (Pittsburgh, PA, USA). The modified BacTiter-Glo Microbial Cell Viability Assay kit (Promega Corporation, Madison, WI, USA) was purchased for the determination of minimum inhibition concentration (MIC). The polymerase chain reaction (PCR) reagents were obtained from Bio-Rad Laboratories (Hercules, CA, USA), and Invitrogen Life Technologies (Carlsbad, CA, USA).

Bacterial culture. Porphyromonas gingivalis ATCC 33277 was purchased from the German Collection of Microorganisms and Cell Cultures (DSMZ; Braunschweig, Germany). The cells were cultured in modified Gifu anaerobic medium (GAM) broth (Nissui, Tokyo, Japan), in an aerobic jar and in the presence of a deoxygenating reagent (AnaeroPack; Mitsubishi Gas Chemical Company, Inc., Tokyo, Japan) for $48 \mathrm{~h}$ at $37^{\circ} \mathrm{C}$. Cell concentration was standardized by measuring optical density at $650 \mathrm{~nm}$ using a Lumetron colorimeter (Photovolt Corp., Indianapolis, IN, USA).

MIC and minimum bactericidal concentration (MBC) assay. MICs were determined with modifications for each organism according to methods described by Cole et al (15). In brief, the appropriate growth medium for $P$. gingivalis was used to prepare 5-ml overnight cultures to an exponential phase. Bacteria were adjusted to a concentration of $4.5 \times 10^{5}$ colony-forming units $/ \mathrm{ml}$, added to various concentrations of antibiotic in 96 -well plates, and incubated at $37^{\circ} \mathrm{C}$ for a period of 18-24 $\mathrm{h}$ in a humidified container. The MIC was defined as the lowest concentration that prevented $50 \%$ growth of cells. MBCs were determined by plating the wells with concentrations of 50-200\% MIC. Following 24-48-h growth, the MBC was determined as the lowest concentration that did not permit visible growth on the surface of the agar. All MIC assays were performed in triplicate.
Scanning electron microscopy (SEM). P. gingivalis cultures were grown to the mid-log phase, and $10 \mathrm{ml}$ cell suspension $\left[1 \times 10^{4}\right.$ cells $/ \mathrm{ml}$ in modified GAM supplemented with $\alpha$-amylase $(12 \mathrm{ng} / \mathrm{ml})$ or pentamidine $(100 \mathrm{ng} / \mathrm{ml})]$ was incubated at $37^{\circ} \mathrm{C}$ for $2 \mathrm{~h}$ prior to collection and fixed in $2.5 \%$ glutaraldehyde. The samples were dehydrated with graded ethanol and t-butanol, dried using the critical point method and coated with gold. Cells were observed under a JSM-6510 LV scanning electron microscope (JEOL, Ltd., Tokyo, Japan).

Determination of differential gene expression. The differential gene expression was determined by quantitative (q) PCR. The bacterial culture $(P$. gingivalis) grown to early exponential phase was adjusted to an optical density 600 of 0.1 and split into two groups. One half was left untreated, while the other half was treated with $\alpha$-amylase $(12 \mathrm{ng} / \mathrm{ml})$ and pentamidine $(100 \mathrm{ng} / \mathrm{ml})$. Following anaerobic incubation for $2 \mathrm{~h}$, the cells were harvested, and total RNA was extracted using TRIzol reagent (Invitrogen Life Technologies, Carlsbad, CA, USA). cDNA was synthesized with $1 \mu \mathrm{g}$ total RNA using the SuperScript II reverse transcriptase (Invitrogen Life Technologies). To identify the expression value of genes associated with hemagglutination, hemolysis, proteolysis, hemin uptake, chromosome replication, energy production, iron storage and oxidative stress, qPCR was performed using specific primers for the selected genes (Table I). The housekeeping gene glyceraldehyde 3-phosphate dehydrogenase (gapA) was used as a control gene. qPCR was conducted using the MiniOpticon Real-Time PCR Detection system (Bio-Rad Laboratories) with a reaction mixture containing $10 \mu \mathrm{l} \mathrm{iQ}$ SYBR Green Supermix (Bio-Rad Laboratories), $1 \mu 1$ cDNA and primers to a final concentration of $250 \mathrm{~nm}$ in a final volume of $20 \mu \mathrm{l}$. To confirm that a single PCR product was amplified, a melting curve analysis was performed under the following conditions: $65^{\circ} \mathrm{C}$ to $95^{\circ} \mathrm{C}$, with a heating rate of $0.2^{\circ} \mathrm{C} / \mathrm{sec}$. All quantifications were normalized to the $P$. gingivalis $16 \mathrm{~S}$ ribosomal RNA gene.

\section{Results}

Minimum inhibition and bactericidal concentration assay. The minimum inhibitory activity against $P$. gingivalis ATCC 33277 cell growth was measured using differential concentrations of $\alpha$-amylase $(2,4,6$ and $8 \mathrm{ng} / \mathrm{ml})$ and pentamidine $(50,75,100$ and $125 \mathrm{ng} / \mathrm{ml})$. The concentrations that prevented $50 \%$ growth of cells observed for $24 \mathrm{~h}$ were considered to be the MIC and were determined as 6 and $100 \mathrm{ng} / \mathrm{ml}$ $\alpha$-amylase and pentamidine, respectively (Fig. 1). The MBC was tested using concentrations of 50-200\% MIC and cells were cultured for 24-48 h. The MBCs were determined as 12 and $100 \mathrm{ng} / \mathrm{ml}$ for $\alpha$-amylase and pentamidine, respectively (Fig. 2). This is the lowest concentration that did not permit visible growth on the surface of the agar, suggesting that $P$. gingivalis cells underwent significant cellular damage.

SEM analysis. SEM results demonstrated that $P$. gingivalis cells treated with $\alpha$-amylase (Fig. 3A) or pentamidine (Fig. 3B) exhibited various stages of lysis. Cellular debris and detached pieces of membrane lay adjacent to the cells. A number of cells were distorted with irregular morphology and loss of cellular 
Table I. Primer sequences used in the current study.

\begin{tabular}{|c|c|c|}
\hline Target gene & Gene identification & Primer sequence $\left(5^{\prime}-3^{\prime}\right)$ \\
\hline \multicolumn{2}{|l|}{$16 \mathrm{~S}$ ribosomal RNA } & F:TGTTACAATGGGAGGGACAAAGGG \\
\hline & & R:TTACTAGCGAATCCAGCTTCACGG \\
\hline \multirow[t]{2}{*}{ gapA } & GAPDH, type I & F:GGCAAACTGACGGGTATGTC \\
\hline & & R:ATGAAGTCGGAGGAAACCAC \\
\hline \multirow[t]{2}{*}{$\operatorname{atp} A$} & ATP synthase subunit A & F:ATCAGGACGGGAAAGACCAC \\
\hline & & R:ACGATGGGGTTGAAAGTGTC \\
\hline \multirow[t]{2}{*}{$\operatorname{cyd} A$} & Cytochrome d ubiquinol oxidase, subunit I & F:TGGATTCTTATCGCCAATGC \\
\hline & & R:ATACGCCCAAAGCAAATACG \\
\hline \multirow[t]{2}{*}{ dnaG } & DNA primase & F:GACACAGGGCTTTCCATCC \\
\hline & & R:GCGAGCAATCTCTTTCTTGG \\
\hline \multirow[t]{2}{*}{ dps } & Dps family protein & F:CAGAAGTGAAGGAAGAGCACGAA \\
\hline & & R:GTAGGCAGACAGCATCCAAACG \\
\hline \multirow[t]{2}{*}{ rbr } & Rubrerythrin & F:TCCACGGCTGAGAACTTGCG \\
\hline & & R:TGCTCGGCTTCCACCTTTGC \\
\hline \multirow[t]{2}{*}{ ftn } & Ferritin & F:CGTGGCGGCGAGGTGAAG \\
\hline & & R:CGGAAGCAGCCCTTACGACAG \\
\hline \multirow[t]{2}{*}{ sodB } & Superoxide dismutase, Fe-Mn & F:GCCAAАCССТCAАCCACААТCTC \\
\hline & & R:GCCATACCCAGCCCGAACC \\
\hline \multirow[t]{2}{*}{ hagA } & Hemagglutinin protein $\mathrm{HagA}$ & F:ACAGCATCAGCCGATATTCC \\
\hline & & R:CGAATTCATTGCCACCTTCT \\
\hline \multirow[t]{2}{*}{ hagB } & Hemagglutinin protein HagB & F:TGTCACTTGACACTGCTACCAA \\
\hline & & R:ATTCAGAGCCAAATCCTCCA \\
\hline \multirow[t]{2}{*}{$\operatorname{rgp} A$} & Arginine-specific cysteine proteinase & F:GCCGAGATTGTTCTTGAAGC \\
\hline & & R:AGGAGCAGCAATTGCAAAGT \\
\hline \multirow[t]{2}{*}{ rgpB } & Arginine-specific cysteine proteinase & F:CGCTGATGAAACGAACTTGA \\
\hline & & R:CTTCGAATACCATGCGGTTT \\
\hline \multirow[t]{2}{*}{$\operatorname{kgp}$} & Lysine-specific cysteine proteinase & F:GCTTGATGCTCCGACTACTC \\
\hline & & R:GCACAGCAATCAACTTCCTAAC \\
\hline
\end{tabular}

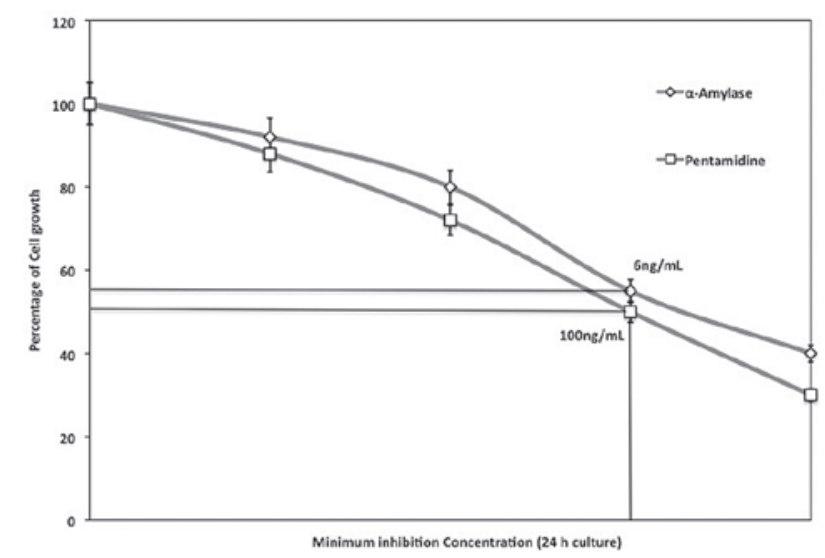

Figure 1. Concentration required to inhibit $50 \%$ growth of $P$. gingivalis indicated the minimum inhibitory concentration of $\alpha$-amylase and pentamidine treatment.

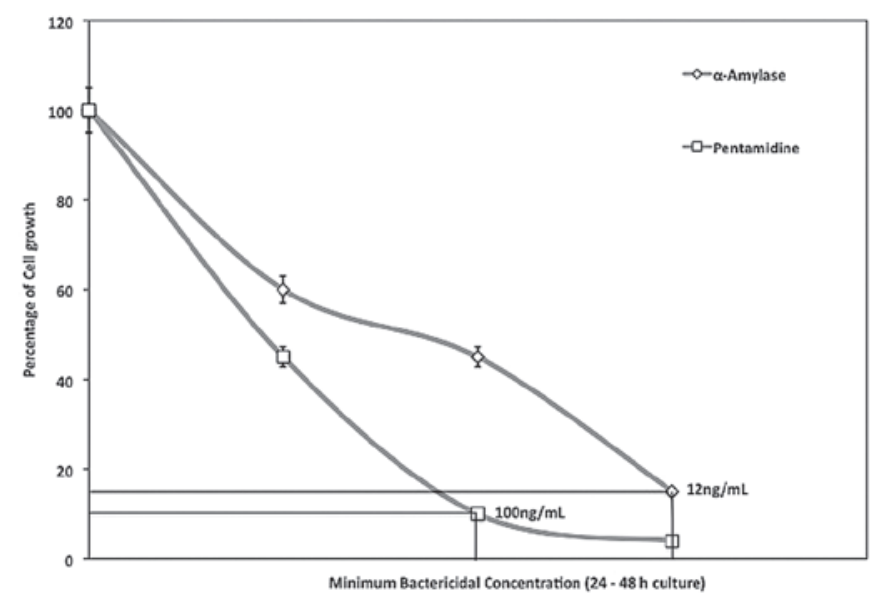

Figure 2. Concentration required to kill $50 \%$. gingivalis was denoted as the minimum bacterial concentration of $\alpha$-amylase and pentamidine treatment. content. In addition, the cells were more closely aggregated and increased numbers of external blebs were present on and around the bacteria compared with those in controls (Fig. 3A).
Similar to $\alpha$-amylase-treated bacteria, pentamidine-treated $P$. gingivalis (Fig. 3B) was also distorted with irregular morphology and presented various stages of lysis with loss 
A

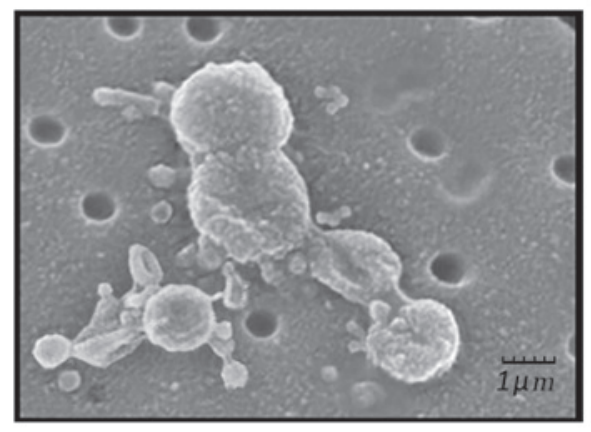

B

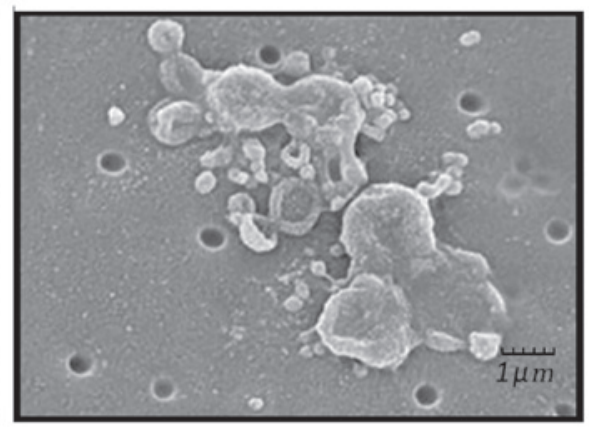

C

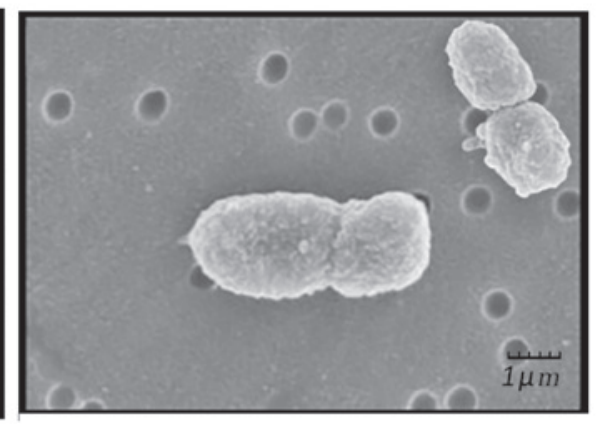

Figure 3. Scanning electron microscopy images presenting the effects of $\alpha$-amylase and pentamidine on $P$. gingivalis. Treatment of $P$. gingivalis with (A) $\alpha$-amylase or $(B)$ pentamidine for $1 \mathrm{~h}$ resulted in evidence of cellular distortion with aggregation of cells and lysis, and detached pieces of membrane lying adjacent to the cells. (C) Untreated cells exhibited a morphology typical of healthy P. gingivalis Gram-negative coccobacilli.

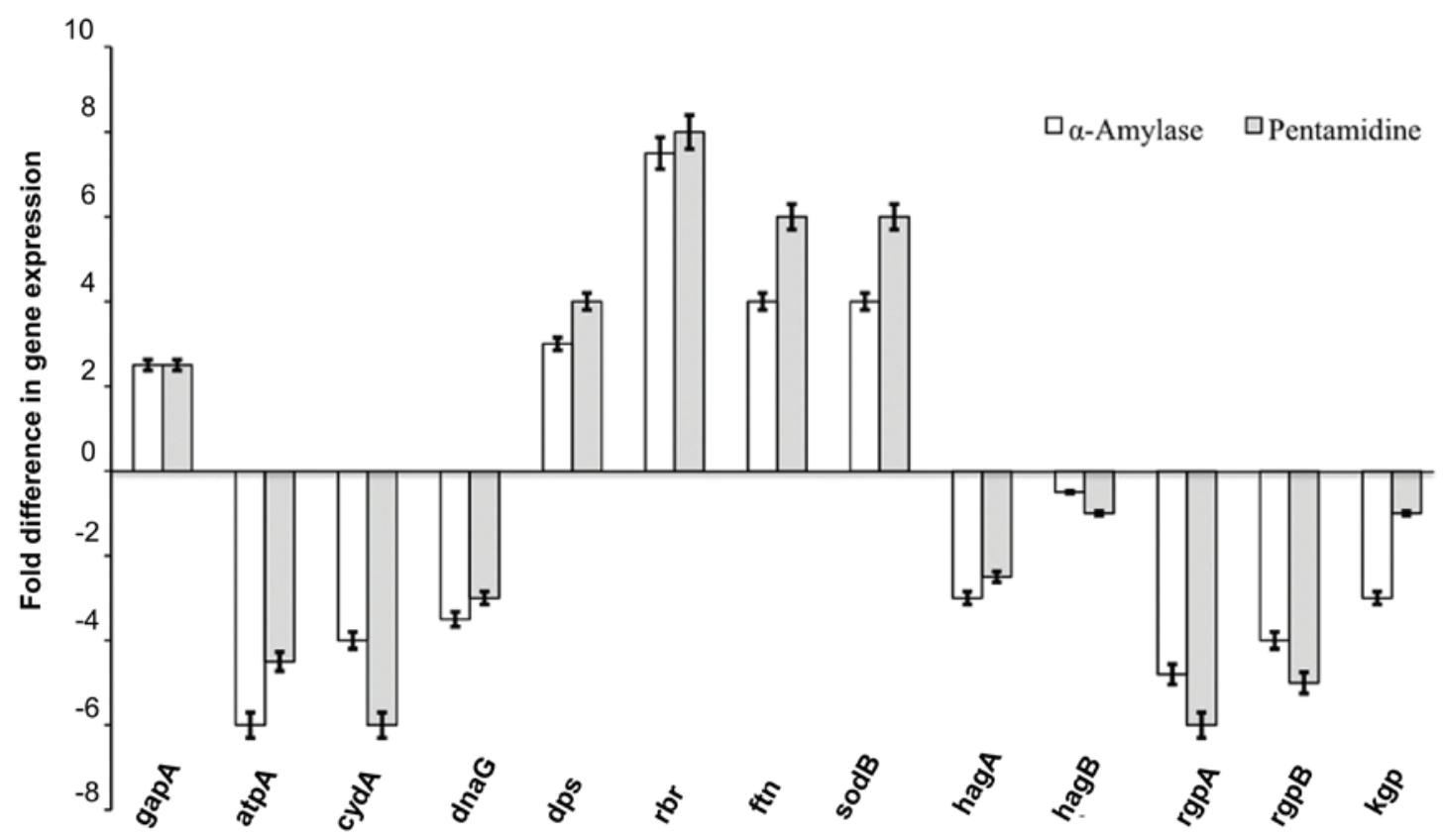

Figure 4. Expression levels of genes associated with hemin uptake, hemagglutination, hemolysis, proteolysis, energy production, chromosome replication, iron storage and oxidative stress. GapA was used as the control gene. Gene expression was measured by quantitative-polymerase chain reaction and normalized to that of the 16S ribosomal RNA gene. The expression level of each gene in the absence of inhibitors ( $\alpha$-amylase and pentamidine) was set as 1 -fold. The results are presented as the mean \pm standard error of three independent experiments.

of intracellular content. Untreated P. gingivalis (Fig. 3C) cells exhibited an external structure typical of a healthy Gram-negative coccobacillus 7 bacterium with multiple blebs present on the cell surface.

Determination of differential gene expression. Following determination of the $\mathrm{MBC}$, differential gene expression was investigated by exposing the culture to the MBC. The genes associated with iron storage (dps, rbr and ftn) and oxidative stress (sodB) were indicated to have upregulated expression levels, while levels of genes encoding gingipains (rgpA, $\operatorname{rgpB}$ and kgp) and hemagglutinins (hagA and hagB) were downregulated by $\alpha$-amylase and pentamidine (Fig. 4). The inhibitors also downregulated the expression of genes associated with energy production (atpA and cydA) and chromosome replication (dnaG). The expression levels of the control gene gapA were not significantly affected.

\section{Discussion}

In the current study, two potent inhibitors of the $P$. gingivalis species ( $\alpha$-amylase and pentamidine) were investigated. These amylases, which are endogenous to saliva and oral mucosa are antimicrobial for $P$. gingivalis, and induce structural damage. $\alpha$-amylase and pentamidine demonstrated significant inhibitory activity against $P$. gingivalis cell growth, and had MICs of 6 and $100 \mathrm{ng} / \mathrm{ml}$, respectively. Similarly, the MBCs of $\alpha$-amylase and pentamidine were determined as 12 and $100 \mathrm{ng} / \mathrm{ml}$, respectively. SEM analysis suggested that the cell membrane structure of bacterial cells was compromised, likely 
resulting from damage caused by the inhibitors. However, the nature and mechanisms of action of the inhibitors remain unclear.

The results of the present study are in agreement with growing evidence that pentamidine kills bacteria in a dose-dependent manner and induces cellular damage. For example, E. coli and $S$. aureus treated with sphingosine, phytosphingosine or dihydrosphingosine exhibit extensive and differential intracellular and extracellular damage (16). Bibel et al (17) also demonstrated that sphinganine (dihydrosphingosine) treatment of $S$. aureus results in ultrastructural damage similar to antibiotic treatment, including lesions of the cell wall, membrane evaginations and leakage. In addition, treatment of Helicobacter pylori with oleic or linoleic acid results in altered morphology, with a disruption of cellular membranes and cell lysis (18). The present study indicates that there may be different mechanisms underlying the actions of different inhibitors. Antimicrobial activity and ultrastructural damage are dependent upon the specific lipid treatment. These data, combined with a previous observation that fatty acids and sphingoid bases exhibit differential activity across bacterial species (19), suggest that the antimicrobial activity of fatty acids and sphingoid bases is a specific interaction that depends upon characteristics of the bacterium and a particular lipid. The current study indicates that the mechanisms for the antimicrobial activity of $\alpha$-amylase and pentamidine against bacteria involve membrane disruption by detergent activity and incorporation of lipids into the bacterial plasma membrane.

Surface-accumulated hemin is transported into bacterial cells so that it can be utilized. To evaluate the effect of $\alpha$-amylase and pentamidine on $P$. gingivalis, the expression levels of selected genes were analyzed to determine whether they were up- or downregulated. The genes associated with iron storage (dps, rbr and ftn) and oxidative stress (sodB) presented upregulated expression levels. Notably, pentamidine increased the level of cell-associated hemin, which suggests that surplus hemin is accumulated on the bacterial cell surface regardless of energy-driven transport in the presence of pentamidine. A small decrease in the level of kgp was observed, the suppressed formation of $\mu$-oxo bisheme may be explained by the fact that $\mathrm{RgpA}$ or $\mathrm{RgpB}$, or the two together, with kgp activity are required by $P$. gingivalis to produce $\mu$-oxo bisheme (20). The formation of $\mu$-oxo bisheme represents an oxidative buffer mechanism for inducing an anaerobic miroenvironment and protects from hemin-mediated cell damage $(20,21)$. Therefore, excessive accumulation of hemin in the vicinity of the bacterial cell surface without formation of $\mu$-oxo bisheme by the bacterium may cause oxidative stress on $P$. gingivalis. This expectation was confirmed by qPCR, which indicated upregulation of the genes involved in oxidative stress, such as dps, rbr, ftn and sodB. An oxidative-stress-like phenomenon is one of the shared downstream events leading to bacterial cell death initiated by bactericidal antibiotics (22). Additionally, during bacterial cell death, genes for energy production, chromosome replication and nucleotide metabolism have been demonstrated to be inactivated (23). Therefore, the observation of the oxidative-stress-like response of $P$. gingivalis and decreased expression of the genes required for ATP synthesis and chromosome replication of the bacterium grown with $\alpha$-amylase and pentamidine in the current study may also support the idea that these inhibitors have a bactericidal effect.

In conclusion, the present study demonstrated that the use of the growth inhibitors $\alpha$-amylase and pentamidine for controlling bacterial infection and aiding the innate immune system, in addition to promoting gene expression, may be an effective strategy for the prevention and treatment of oral cavity infection. Following comparison of these two inhibitors, pentamidine was demonstrated to be more effective at inhibiting the growth of $P$. gingivalis cells. However, further investigation is required to investigate its suitability for use in humans.

\section{References}

1. Darveau RP, Tanner A and Page RC: The microbial challenge in periodontitis. Periodontol 2000 14: 12-32, 1997.

2. Eke PI, Dye BA, Wei L, et al; CDC Periodontal Disease Surveillance Workgroup: Prevalence of periodontitis in adults in the United States: 2009 and 2010. J Dent Res 91: 914-920, 2012.

3. Hutter G, Schlagenhauf U, Valenza G, et al: Molecular analysis of bacteria in periodontitis: evaluation of clone libraries, novel phylotypes and putative pathogens. Microbiology 149: 67-75, 2003.

4. Hajishengallis G, Liang S, Payne MA, et al: Low-abundance biofilm species orchestrates inflammatory periodontal disease through the commensal microbiota and complement. Cell Host Microbe 10: 497-506, 2011

5. Holt SC, Kesavalu L, Walker S and Genco CA: Virulence factors of Porphyromonas gingivalis. Periodontol 2000 20: 168-238, 1999.

6. Lamont RJ and Jenkinson HF: Subgingival colonization by Porphyromonas gingivalis. Oral Microbiol Immunol 15: 341-349, 2000.

7. Gorr SU: Antimicrobial peptides in periodontal innate defense. Front Oral Biol 15: 84-98, 2012.

8. Gupta R, Gigras P, Mohapatra H, et al: Microbial $\alpha$-amylases: a biotechnological perspective. Process Biochem 38: 1599-1616, 2003.

9. Rajagopalan G and Krishnan C: Alpha-amylase production from catabolite derepressed Bacillus subtilis KCC103 utilizing sugarcane bagasse hydrolysate. Bioresour Technol 99: 3044-3050, 2008.

10. Pandey A, Nigam P, Soccol CR, et al: Advances in microbial amylases. Biotechnol Appl Biochem 31 (Pt 2): 135-152, 2000.

11. Burchmore RJ, Ogbunude PO, Enanga B and Barrett MP: Chemotherapy of human African trypanosomiasis. Curr Pharm Des 8: 256-267, 2002

12. Wilson WD, Tanious F, Mathis A, et al: Antiparasitic compounds that target DNA. Biochimie 90: 999-1014, 2008.

13. Pearson RD and Hewlett EL: Pentamidine for the treatment of Pneumocystis carinii pneumonia and other protozoal diseases. Ann Intern Med 103: 782-786, 1985.

14. Charpentier TH, Wilder PT, Liriano MA, et al: Divalent metal ion complexes of S100B in the absence and presence of pentamidine. J Mol Biol 382: 56-73, 2008.

15. Cole AM, Weis $P$ and Diamond G: Isolation and characterization of pleurocidin, an antimicrobial peptide in the skin secretions of winter flounder. J Biol Chem 272: 12008-12013, 1997.

16. Fischer CL, Walters KS, Drake DR, et al: Sphingoid bases are taken up by Escherichia coli and Staphylococcus aureus and induce ultrastructural damage. Skin Pharmacol Physiol 26: 36-44, 2013.

17. Bibel DJ, Aly R, Shah S and Shinefield HR: Sphingosines: antimicrobial barriers of the skin. Acta Derm Venereol 73: 407-411, 1993.

18. Khulusi S, Ahmed HA, Patel P, Mendall MA and Northfield TC: The effects of unsaturated fatty acids on Helicobacter pylori in vitro. J Med Microbiol 42: 276-282, 1995.

19. Fischer CL, Drake DR, Dawson DV, et al: Antibacterial activity of sphingoid bases and fatty acids against Gram-positive and Gram-negative bacteria. Antimicrob Agents Chemother 56: 1157-1161, 2012. 
20. Smalley JW, Birss AJ, Szmigielski B and Potempa J: The HA2 haemagglutinin domain of the lysine-specific gingipain (Kgp) of Porphyromonas gingivalis promotes micro-oxo bishaem formation from monomeric iron(III) protoporphyrin IX. Microbiology 152: 1839-1845, 2006.

21. Lewis JP, Dawson JA, Hannis JC, Muddiman D and Macrina FL: Hemoglobinase activity of the lysine gingipain protease (Kgp) of Porphyromonas gingivalis W83. J Bacteriol 181: 4905-4913, 1999.
22. Wright GD: On the road to bacterial cell death. Cell 130: 781-783, 2007.

23. Asakura Y and Kobayashi I: From damaged genome to cell surface: transcriptome changes during bacterial cell death triggered by loss of a restriction-modification gene complex. Nucleic Acids Res 37: 3021-3031, 2009. 